\title{
FORCAST: A Mid-Infrared Camera for SOFIA
}

\author{
T. L. Herter ${ }^{1,6}$, J. D. Adams ${ }^{2}$, G. E. Gull ${ }^{1}$, J. Schoenwald ${ }^{1}$, \\ L. D. Keller ${ }^{3}$, B. E. Pirger ${ }^{1}$, C. P. Henderson ${ }^{1}$, G. J. Stacey ${ }^{1}$, T. Nikola ${ }^{1}$, \\ J. M. De Buizer ${ }^{4}$, W. D. Vacca ${ }^{4}$ and K. Ennico ${ }^{5}$ \\ ${ }^{1}$ Department of Astronomy, Cornell University \\ Ithaca, NY 14853, USA \\ ${ }^{2}$ Universities Space Research Association \\ NASA/Armstrong Flight Research Center \\ 2825 East Avenue P, Palmdale, CA 93550, USA \\ ${ }^{3}$ Department of Physics \& Astronomy \\ Ithaca College, Ithaca, NY 14850, USA \\ ${ }^{4}$ Universities Space Research Association \\ NASA Ames Research Center \\ MS 211-3, Moffett Field, CA 94035, USA \\ ${ }^{5}$ NASA Ames Research Center \\ Moffett Boulevard, Moffett Field, CA 94035, USA \\ ${ }^{6}$ tlh10@cornell.edu \\ Received April 16, 2018; Accepted June 27, 2018; Published July 24, 2018
}

\begin{abstract}
We describe the Faint Object infraRed CAmera for the SOFIA Telescope (FORCAST) which is presently operating as a facility instrument on the Stratospheric Observatory For Infrared Astronomy (SOFIA). FORCAST provides imaging and moderate resolution spectroscopy capability over the $5-40 \mu \mathrm{m}$ wavelength range. In imaging mode, FORCAST has a $3.4^{\prime} \times 3.2^{\prime}$ field-of-view with $0.768^{\prime \prime}$ pixels. Using grisms, FORCAST provides long-slit low-resolution $(R=100-300)$ and short-slit, cross-dispersed mediumresolution spectroscopic modes $(R=800-1200)$ over select wavelengths. Preceded by both Spitzer and Herschel, the discovery phase space for FORCAST lies in providing unique photometric bands and/or spectroscopic coverage, higher spatial resolution and exploration of the brightest sources which typically saturate space observatories.
\end{abstract}

Keywords: SOFIA (FORCAST), airborne, imaging, spectroscopy, mid-infrared.

\section{Introduction}

The Faint Object infraRed CAmera for the SOFIA Telescope (FORCAST) is a wide-field camera operating in the infrared from $5-40 \mu \mathrm{m}$. First light on SOFIA was achieved with FORCAST on May 26, 2010. Near diffraction-limited-performance was obtained at the longest wavelengths of FORCAST. Two observatory characterization flights (OCF2 and OCF3) were flown in November 2011 and three "short" science flights in December 2011 (Young et al., 2012; Herter et al., 2012). The short science flights offered an opportunity to obtain science data while informally commissioning a number of operating modes (Adams et al., 2012a; De Buizer et al., 2012; Shuping et al., 2012; Salgado et al., 2012; Harvey et al., 2012; Nikola et al., 2012; Hirsch et al.,

\footnotetext{
${ }^{6}$ Corresponding author.

This is an Open Access article published by World Scientific Publishing Company. It is distributed under the terms of the Creative Commons Attribution 4.0 (CC-BY) License. Further distribution of this work is permitted, provided the original work is properly cited.
} 
2012). A "basic" science program of ten flights with FORCAST were flown in May-June 2012 in support of the general community followed by formal instrument commissioning and acceptance which occurred over a series of flights from AprilJuly 2013. Since this time FORCAST has been operating as a facility instrument open to the general community and has been used for a variety of science projects. A few examples include planetary science (Fletcher et al., 2017), protoplanetary nebula (Arneson et al., 2017), protostars (Adams et al., 2012a; Hirsch et al., 2012; Zhang et al., 2013; De Buizer et al., 2017), nova (Gehrz et al., 2018), evolved stars (Gordon et al., 2018; Hankins et al., 2018; Materese et al., 2017), and galaxies (Fuller et al., 2016; Lopez-Rodriguez et al., 2018).

In the subsequent sections we describe the instrument science objectives, optical design, thermal and mechanical design, detectors, electronics, operations, and performance of FORCAST.

\section{Instrument Science Objectives}

Dust heated via UV and optical photons in regions of star formation, evolved stars, and active galactic nuclei (AGN) emits mainly in the infrared. The composition, spatial distribution and temperature of this dust are of general interest to understand source properties, e.g. luminosity, heating sources, dust production/destruction, etc. Typically dust in stellar and HII region environments shows thermal emission that peaks in the mid-infrared spectrum which is uniquely accessible to airborne and spacebased platforms.

Both molecules and grains possess unique midinfrared $(5-40 \mu \mathrm{m})$ spectral signatures, which are very useful for establishing their identity and abundance, studying their environments, and their conditions of formation and destruction. Hydrocarbons such as polycyclic aromatic hydrocarbons (PAHs) have strong spectral features over the $3.3-16 \mu \mathrm{m}$ range, and these are commonly seen in regions of star formation (in emission and sometimes in absorption). For instance, the 6-9 $\mathrm{m}$ region contains features that arise from ionized PAHs that provide diagnostics of radiation environments. Interstellar mineral grains also have a variety of spectral features in the 5-40 $\mu \mathrm{m}$ wavelength region; spectroscopic observations can reveal how grains are processed as they migrate to circumstellar environments around young stars.

There are a number of broad mid-IR atomic lines found in high velocity environments (widths
$100-1000 \mathrm{~km} / \mathrm{s}$ ) such as stellar ejecta and around AGNs and massive star clusters which are used as diagnostics of abundances and excitations. $R \sim 1000$ spectroscopy is ideal for these lines because the resolution is well-matched to their line widths for optimal sensitivity. For example, in planetary nebulae (PNe), strong mid-IR excitation lines such as HI $\mathrm{Br} \alpha, 4.49 \mu \mathrm{m}[\mathrm{Mg}$ IV] $, 4.53 \mu \mathrm{m}[\mathrm{Ar} \mathrm{VI}], 5.61 \mu \mathrm{m}[\mathrm{Mg}$ $\mathrm{V}], 6.99 \mu \mathrm{m}[\mathrm{Ar} \mathrm{II}], 7.65 \mu \mathrm{m}[\mathrm{Ne} \mathrm{VI}]$, and $8.99 \mu \mathrm{m}[\mathrm{Ar}$ III] are more robust abundance indicators, compared to UV and optical diagnostic lines, as these mid-IR lines are less sensitive to extinction and temperature dependence. Mid-IR spectroscopic excitation lines in novae and supernovae (SNe) show heavier elements, such as Al, Si, Ne and $\mathrm{S}$ in the $3-38 \mu \mathrm{m}$ region and $\mathrm{Ni}$ and Co at $6-16 \mu \mathrm{m}$, respectively. The $\mathrm{Ni}$ and the 20 $30 \mu \mathrm{m} \mathrm{Fe}$ lines are used to trace production of irongroup elements in SNe. See Ennico et al. (2006) for a more extensive discussion of the science justification of the FORCAST spectroscopy modes.

\section{Instrument Overview}

FORCAST (Fig. 1) is a dual-channel, mid-infrared camera and spectrograph designed and built at Cornell University. An enabling technology for FORCAST is the use of two $256 \times 256$ Si BlockedImpurity-Band (BIB) focal plane arrays (Sec. 4.3). Observations can be made through either of the

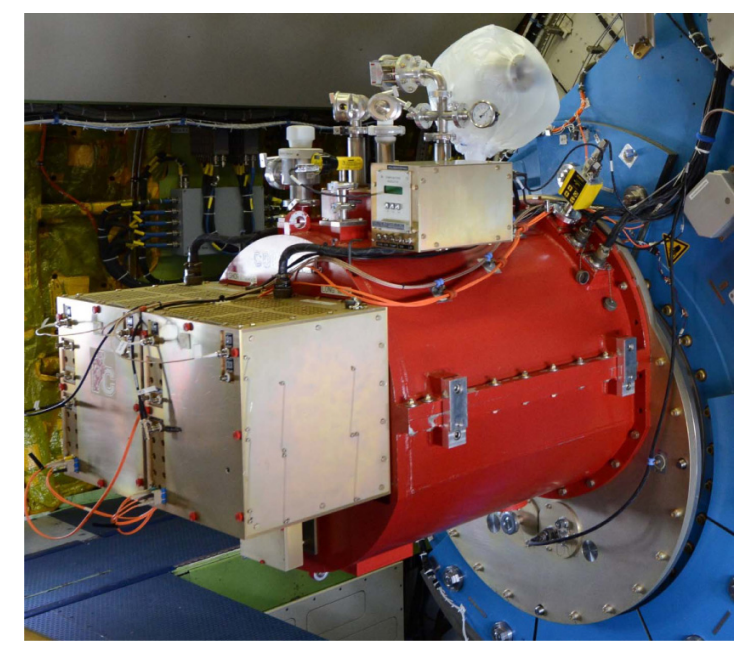

Fig. 1. FORCAST (red) bolted to SOFIA telescope mounting flange (blue). The electronics for the two arrays are contained in the two metal boxes on the left. The small metal box on top operates temperature sensors located inside the dewar. The total mass as seen here including the mounting plate, electronics, and cryogens is $431 \mathrm{~kg}$. Including the electronics boxes, the instrument is $1.1 \mathrm{~m}$ long. 
Table 1. FORCAST configurations. ${ }^{\text {a }}$

\begin{tabular}{lcccc}
\hline Mode $^{\mathrm{b}}$ & $\begin{array}{c}\text { Pixel } \\
\left({ }^{\prime \prime}\right)\end{array}$ & $\begin{array}{c}\mathrm{FoV}^{\mathrm{c}} \\
\left({ }^{\prime \prime}\right)\end{array}$ & $\begin{array}{c}\lambda \text { Range }^{\mathrm{d}} \\
(\mu \mathrm{m})\end{array}$ & $\lambda / \Delta \lambda$ \\
\hline SWC Img & 0.768 & $204 \times 191$ & $5.4 \ldots 25.3$ & $7-70$ \\
LWC Img & 0.768 & $204 \times 191$ & $8.6 \ldots 37.1$ & $5.5-47$ \\
Dual Img & 0.768 & $204 \times 191$ & $5.4 \ldots 37.1$ & \\
G063 & 0.768 & $4.7 \times 191$ & $4.9-8.0$ & 180 \\
XG063b & 0.768 & $2.4 \times 11.2$ & $4.9-8.0$ & 1170 \\
G111 & 0.768 & $4.7 \times 191$ & $8.4-13.7$ & 130 \\
G227 & 0.768 & $4.7 \times 191$ & $17.6-27.7$ & 110 \\
G329 & 0.768 & $4.7 \times 191$ & $28.7-37.1$ & 160 \\
\hline
\end{tabular}

Notes: ${ }^{a}$ Broadband imaging and spectroscopic modes. See SOFIA Observer's Handbook for the available configurations. ${ }^{b}$ Broadband imaging or grism spectroscopy. SWC and LWC are the short and long wavelength channels.

${ }^{\mathrm{c}} \mathrm{FoV}=$ Field of view. A $2.4 \times 191$ slit is also available.

${ }^{\mathrm{d}}$ For nominal imaging filters see Table 4.

two channels individually or, by use of a dichroic internal to FORCAST, with both channels simultaneously. Bandpass filters are individually selectable for each channel. A grism and slit combination can be chosen for low- and medium-resolution spectroscopy. The grism suite consists of six grisms: four monolithic Si grisms and two KRS-5 grisms which allow long-slit low-resolution $(R=100-300)$ and short-slit, cross-dispersed high resolution spectroscopic modes $(R=800-1200)$ over selected wavelengths. The available instrument configurations are summarized in Table 1.

The instrument has slightly different magnifications in the $x$ and $y$ directions, which in imaging mode, after post-processing, results in a $3.4^{\prime} \times 3.2^{\prime}$ instantaneous field-of-view with square pixels of $0.768^{\prime \prime}$. The orientation of this field on the sky depends the field rotation at the time of the observation.

\section{Instrument Design}

\subsection{Optical design}

The short-wavelength channel (SWC) and longwavelength channel (LWC) are functionally identical in design. Reflective optics consisting of gold-coated, diamond-turned aluminum mirrors make the two systems wavelength independent leaving the defining characteristics as the filters and detectors. The requirements for the optical subsystems are threefold: (1) re-image the telescope focal plane to the detector focal plane at the proper scale, (2) provide a cold baffle so that the instrument sees only telescope and sky, and (3) provide a collimated

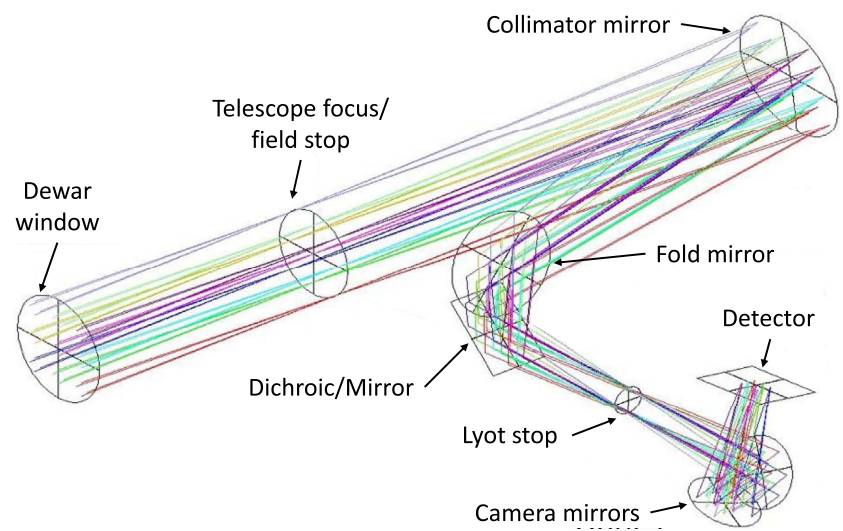

Fig. 2. The optical path of the short-wavelength FORCAST channel. Lights enters through the Dewar window on the left, reflects from a collimator, fold-mirror, and dichroic before being focus onto the detector by the camera assembly shown on the lower-right (see Sec. 4.1 for details). A six-position aperture wheel is located at the field stop and two six-position filter wheels are located at the Lyot stop, one on either side.

beam for the placement of bandpass filters and grisms.

The basic design principles that determine the properties of the optical elements are straightforward. The telescope is focused onto the instrument field stop which is located at the focus of a collimator. The collimator f-number matches that of the telescope $\left(f_{T}^{\#}=19.7\right)$ while the focal length of the collimator, $f_{\text {col }}$, is set by the desired Lyot stop size $(<25 \mathrm{~mm})$. Choosing $f_{\text {col }}=450 \mathrm{~mm}$ yields a Lyot stop diameter of $\sim 23 \mathrm{~mm}$. For a pixel size, $x_{p}$, sampling $p$ pixels across the diffraction disk $\left(\theta_{T}=1.22 \lambda / D_{T}\right)^{(\text {a) }}$ at wavelength, $\lambda$, the f-number of the detector camera is given by:

$$
f_{\text {cam }}^{\#}=p \frac{x_{p}}{1.22 \lambda} \text {. }
$$

Sampling two pixels across the diffraction disk at $15 \mu \mathrm{m}, f_{\text {cam }}^{\#}=5.46$ and $0.75^{\prime \prime}$ pixels for the $50 \mu \mathrm{m}$ pixels of the detector. At the telescope focal plane a pixel maps to:

$$
x_{T}=x_{p} \frac{f_{T}^{\#}}{f_{\text {cam }}^{\#}}=180 \mu \mathrm{m},
$$

so that for imaging the field stop size is $46.2 \mathrm{~mm}$ ( 256 pixels). The estimates above are close to actual values but differ slightly because of both the breakdown of the Gaussian optics approximation and optimization of the design.

\footnotetext{
${ }^{a}$ Another choice could have been $\theta_{T}=\lambda / D_{T}$, the full-widthhalf-maximum (FWHM) of the Airy disk but we opted for a larger field-of-view since the expected in-flight PSF would be well-sampled with the adopted pixel scale.
} 
Figure 2 shows the optical path for the SWC. Light from the telescope enters the dewar through a $7.6 \mathrm{~cm}$ (3.0 in) diameter CsI window, passes through an $\mathrm{LN}_{2}$ cold stop (not shown) and is focused at the field stop where a six position aperture wheel is located. The wheel holds an imaging field stop, slits used for spectroscopy, and a collection of field masks for instrument characterization.

The light passes to an off-axis, hyperbolic collimator mirror before striking a first fold-mirror, which redirects the light into the LHe-cooled portion of the cryostat. $^{(b)}$ After entering the LHe bench, the incoming beam reaches a "dichroic" slide, which includes an open position, a mirror, and two dichroics. The open position of the slide passes the beam to a second fold-mirror, which sends the beam to the LWC, while the mirror position redirects the light to the SWC. The dichroics, which were fabricated by Barr UK, are silicon with CdSe and/or PbTe multilayer coats. They reflect light below $26 \mu \mathrm{m}$ to the SWC and pass light from $26-40 \mu \mathrm{m}$ to the LWC.

After the dichroic, the light passes through a Lyot stop. The collimator images the secondary mirror of the telescope onto the Lyot stop providing a cold baffle which defines the entrance pupil of the telescope. Filter wheels with six slots each are located on either side of the Lyot stop, allowing combinations of up to 10 separate filters per channel. ${ }^{\text {(c) }}$ After passing through the filter assembly, the incoming beam enters a camera block that consists of two offaxis concave mirrors, one hyperbolic and the other ellipsoidal, which combine to focus the light onto the focal plane array. A movable silicon lens (not shown) can be inserted into the beam between the last mirror and the detector to produce an image of the Lyot stop onto the array, facilitating alignment of the collimator mirror with the telescope optical axis.

Figure 3 shows the computed image quality and image distortion at the focal plane. The geometric aberrations are significantly less that the diffraction spot size at $15 \mu \mathrm{m}$ and the distortion is (at worst) $\sim 1 \%$. In practice, a pinhole grid, placed in the aperture wheel, was used to determine the distortion corrections that are applied as part of normal calibration (Adams et al., 2012b).

Grisms are used for low and medium resolution spectroscopy. Slits are located in the aperture wheel

\footnotetext{
$\overline{{ }^{\mathrm{b}} \text { The collimator focal length must be long enough }}$ to avoid collisions between the incoming beam to the collimator and the fold-mirror.

${ }^{\mathrm{c}}$ The current filter set with transmission curves is available in the SOFIA Observer's Handbook.
}

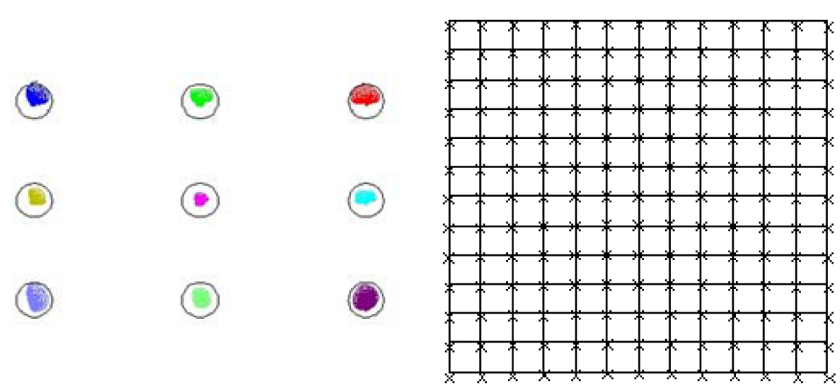

Fig. 3. Left: Spot diagrams calculated in the FORCAST focal plane in a 9 point square grid with the outer 8 spots at the edge of the field. Spots are shown for the center and the edges of the array. The circles represent the Airy disk at $15 \mu \mathrm{m}$. Right: Illustration of the image distortion. In each $12 \times 12$ grid, representing the full focal plane, a box represents just over 21 pixels. Distortion is at worst $1.1 \%$ or $\sim 3$ pixels relative to the center of the detector array; at least $1 / 2$ of the array area is at $<1$ pixel of distortion.

at the field stop and grisms are in the filter wheel. For medium resolution spectroscopy, two grisms are cross-dispersed with one in each wheel. ${ }^{(d)}$

\subsection{Thermal and mechanical}

Figures 4 and 5 show two schematic top-level views of FORCAST. The instrument is cryogenically-cooled, open-cycle liquid nitrogen $\left(\mathrm{LN}_{2}\right)$ /liquid helium (LHe) system. The cryogen cans are capped-off

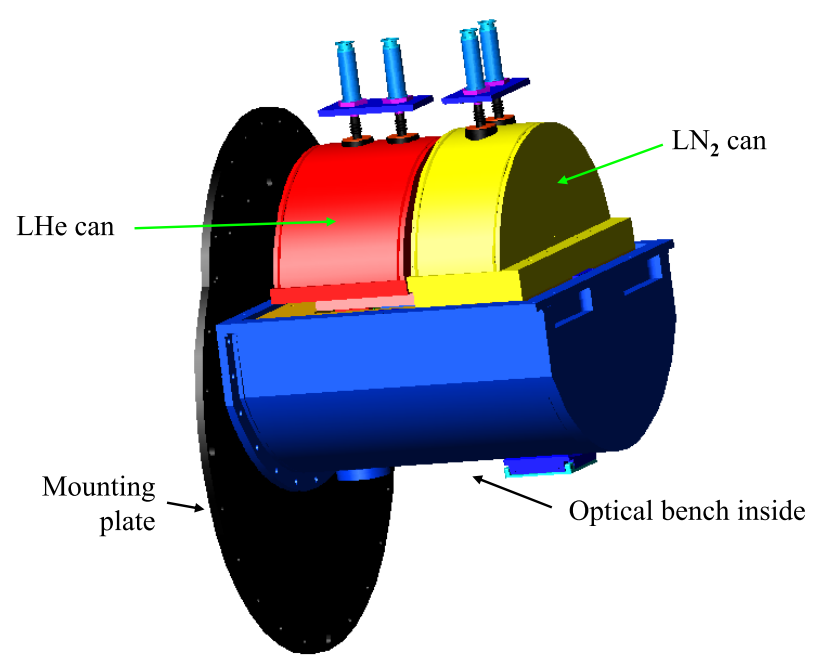

Fig. 4. Simplified solid model view of FORCAST showing the dewar mounting plate, liquid helium reservoir, liquid nitrogen reservoir, and the vacuum shell covering the optics bench. Light enters from the left through a hole (not seen) in the mounting plate. The volumes of the $\mathrm{LN}_{2}$ and LHe reservoirs are 20 and 301 , respectively.

\footnotetext{
The installed grisms and their parameters are available in the SOFIA Observer's Handbook.
} 


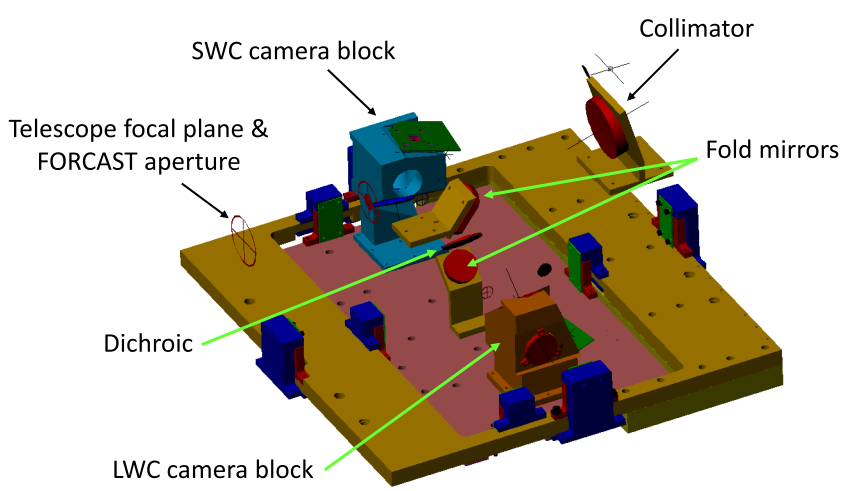

Fig. 5. CAD model showing the $\mathrm{LN}_{2}$ (brownish-orange) and LHe-cooled (pink) optical benches show the location of the some of the optical components (red). See Sec. 4.1 and Fig. 2 for a description of the optical path.

with pressure-relief valves after filling. The dichroic, filters and detectors are maintained at LHe temperatures. The field stop and collimator are kept at $\mathrm{LN}_{2}$ temperatures. The overall instrument volume is set by the size and layout of the optical components. The cryostat is about $0.75 \mathrm{~m}$ long and $0.79 \mathrm{~m}$ in diameter making the $1 / 4$-inch thick outer shell very stiff.

Radiation shielding is provided by mylar multilayer insulation (MLI). Double-sided tape attaches a layer to the inside of the vacuum shell while roughly 10 layers of MLI cover the $\mathrm{LN}_{2}$ shield. No "wedding veil" separates the MLI layers which are aluminized only on one side. Testing on previous dewars showed when the MLI is set up this way the infrared emissivity is about $0.02( \pm 0.05)$. For design purposes we assumed a conservative bare aluminum emissivity of 0.2 . Four $(\mathrm{L} \times \mathrm{W} \times \mathrm{T}=2 \times 2 \times$ 0.0625 inch) $\mathrm{G} 10$ fiberglass tabs attach the $\mathrm{LN}_{2}$ work surface to the vacuum shell and four $(2 \times 1.5 \times 0.0625$ inch $)$ tabs connect the LHe work surface to the $\mathrm{LN}_{2}$ work surface. All motors (made by Phytron) are mounted on the $\mathrm{LN}_{2}$ work surface and G10 hollow-cylinders (0.0302 inch $^{2}$ cross-section) serve as drive shafts to the filter wheels and dichroic slide reducing heat conduction into $\mathrm{LHe}$ stage. Additional heat input comes from wires and the entrance window for both stages. Wires provide a significant load into the LHe reservoir; these consist of stainless coax for DC biases, clocking and signal readout and a copper return for each array.

Table 2 shows the estimated division of heat loads inside the cryostat. These values are based on intermediate testing of the cryostat during assembly and (rough) radiative or conduction calculations using the physical properties of the materials. For
Table 2. Cryostat heat loads.

\begin{tabular}{lcc}
\hline Component & $\begin{array}{c}\mathrm{LN}_{2} \text { Load } \\
(\mathrm{W})\end{array}$ & $\begin{array}{c}\text { LHe Load } \\
(\mathrm{mW})\end{array}$ \\
\hline Radiation & 6.8 & 70 \\
G10 tabs & 0.7 & 74 \\
Wires & 1.0 & 120 \\
Shafts & 0.0 & 13 \\
Window & 2.4 & 20 \\
Total Load & 11 & 297 \\
\hline
\end{tabular}

Notes: ${ }^{\text {a }}$ These loads imply $4.2 \mathrm{hr} / \mathrm{l}$ and $2.4 \mathrm{hr} / 1$ for the $\mathrm{LN}_{2}$ and LHe cryogens, respectively.

the $\mathrm{LN}_{2}$ stage, radiative input from the shell and window dominate. For the LHe stage the heat load is more distributed with the single largest contributor being the two copper return lines for the detector which account for 80 of the $120 \mathrm{~mW}$ allocated to wiring. The LHe window load includes $77 \mathrm{~K}$ radiation through the hole into the LHe volume.

The volume of each cryogen reservoir was then set to provide a hold time longer than three days, obviating the need for weekend servicing on the airplane. The design process can be slightly iterative since the size of the cryogen cans does influence the heat load into the cryostat. The final cryogen volumes are 20 and 301 for the $\mathrm{LN}_{2}$ and LHe cryogen cans, respectively.

FORCAST is equipped with a calibration box that is design to provide flat fields. The box is fixed to the FORCAST mounting plate just below the window and extends into the SOFIA Nasmyth tube when FORCAST is installed. A Peltier thermoelectric heater serves as a flux source. This source is imaged to the FORCAST pupil using a planoconvex, KRS-5 lens. The optical beam is folded with a motorized, flat mirror into the FORCAST window. During normal telescopic observations, this flip mirror is rotated down with a stepper motor to cover the calibration box lens and allow FORCAST to view the telescope. The temperature of the Peltier source is set from the FORCAST control PC through a serial link to the Peltier controller. Typically, $\Delta \mathrm{T}=20^{\circ} \mathrm{C}$ between the reference and the source. This temperature differential results in a large signal $(\mathrm{S} / \mathrm{N}>100)$ on the FORCAST detectors at the nominal operating pixel well depth. More detail on the calibration box hardware can be found in Adams et al. (2010).

The collimator mirror of FORCAST is mounted on a two-axis tipping stage. G10 rods go from drive 
screws on the stage to the outside of the dewar through compression-sealed O-rings, allowing easy alignment of FORCAST to the secondary mirror of the telescope. Once the pupils are aligned, the rods are pulled slightly back from the mirror assembly (but still held captive) to eliminate a thermal conduction path through the rods.

\subsection{Detectors}

FORCAST leverages BIB array technology (Herter et al., 1998; Adams et al., 2004). As:Si and Sb:Si BIBs fabricated by DRS Technologies provide wavelength coverage from approximately $5-25 \mu \mathrm{m}$ and 20 $40 \mu \mathrm{m}$, respectively. At design time, the available $256 \times 256$ arrays coupled with the projected seeing limits of SOFIA in the infrared $\left(\sim 1.5^{\prime \prime}\right)$ allowed for Nyquist sampling while achieving a field-of-view of over roughly $3.0^{\prime}$. Table 3 shows the nominal properties of the arrays. A notable feature of the arrays is a switchable capacitance for the unit cell ("lo-cap" and "hi-cap") which allows a factor of ten change in dynamic range.

The formalism for discussing the performance of BIBs (Herter, 1994) is similar to that for extrinsic photoconductors (EXPC) with the exception that the BIBs have gain and an associated gain dispersion, $\beta$, defined by $\beta=\left\langle G^{2}\right\rangle /\langle G\rangle^{2}$. In the unity gain regime $(\beta=1)$, the noise characteristics of BIBs

Table 3. Detector properties.

\begin{tabular}{lccc}
\hline Specification & Si:As & Si:Sb & Units \\
\hline Wavelength Range & $5-25$ & $25-40$ & $\mu \mathrm{m}$ \\
Array Size & $256 \times 256$ & $256 \times 256$ & pixels \\
Pixel Size & 50 & 50 & $\mu \mathrm{m}$ \\
Filling factor & $>95$ & $>95$ & $\%$ \\
Nominal bias & 1.5 & 1.25 & volts $^{-}$ \\
Read Noise (lo-cap) & 250 & 250 & $\mathrm{e}^{-}$ \\
Well Depth (lo-cap) & $1.6 \times 10^{6}$ & $1.6 \times 10^{6}$ & $\mathrm{e}^{-}$ \\
Read Noise (hi-cap) & 2400 & 2400 & $\mathrm{e}^{-}$ \\
Well Depth (hi-cap) & $1.6 \times 10^{7}$ & $1.6 \times 10^{7}$ & $\mathrm{e}^{-}$ \\
Dark current & $7 \times 10^{4}$ & $5 \times 10^{5}$ & $\mathrm{e}^{-} / \mathrm{s}$ \\
$\beta G^{\mathrm{a}}$ & $\sim 1.3$ & $\sim 1.5$ & \\
$\eta G^{\mathrm{b}}$ & 30 & 30 & $\%$ \\
Pixel FoV & 0.768 & 0.768 & $\operatorname{arcsec}$ \\
Array FoV $^{\mathrm{c}}$ & $3.4 \times 3.2$ & $3.4 \times 3.2$ & $\operatorname{arcmin}$ \\
\hline
\end{tabular}

Notes: ${ }^{\mathrm{a}} \beta G=N^{2} / S$ where $N$ and $S$ are the noise and signal.

${ }^{\mathrm{b}}$ Average quantum efficiency (QE). The detective QE (DQE) is $\eta / \beta=\eta G / \beta G$.

${ }^{\mathrm{c}}$ Field of View (FoV) after distortion correction. are to first order like that of photovoltaic devices. At higher biases (and gains greater than one) there is an increased noise due to gain dispersion. Applying this terminology to photovoltaic and EXPC detectors, for photovoltaics $\beta=1$ and $G=1$, while for EXPCs $\beta=2$ due to generation-recombination noise.

The noise, $N$, in a BIB detector is a combination of photon noise, dark current noise, and read noise. For the detector collecting (quasi-monochromatic) photons of energy $h \nu$ over a time $t$, the noise is:

$$
N^{2}=\frac{P_{B}+P_{S}}{h \nu} \frac{\eta}{\beta}(\beta G)^{2} t+\beta_{d} G_{d} i_{d} t+R_{N}^{2},
$$

where $P_{B}$ and $P_{S}$ are the radiant power per pixel due to the background and the source, respectively, $\eta$ is the quantum efficiency, $G$ is the photoconductive gain, $R_{N}$ is the readout noise, $i_{d}$ is the dark current, $G_{d}$ is the gain of the dark current, and $\beta_{d}$ is the gain dispersion of the dark current. In general, we expected $\beta_{d} G_{d} \sim \beta G$ but measurements of the current focal planes indicate that while $\beta G$ is near unity for photons (Table 3), it is about 7 and 15 for the Si:AS and $\mathrm{Si}: \mathrm{Sb}$ detectors, respectively, at nominal bias.

Let $S$, the signal, be the number of collected electrons generated by radiation from the source of interest:

$$
S=\frac{P_{S}}{h \nu} \frac{\eta}{\beta}(\beta G) t .
$$

When the fluctuations in the radiation power dominate dark current noise and read noise (background limited conditions), the signal-to-noise ratio is dependent upon the ratio, $\eta / \beta$, which we call the detective quantum efficiency. Assuming this case and that $P_{B} \gg P_{S}$, the sensitivity is given by

$$
P_{S}=\frac{S}{N}\left[\frac{P_{B} h \nu}{(\eta / \beta) t}\right]^{1 / 2} \text {. }
$$

It is not possible to measure $\eta, \beta$ and $G$ separately but only the quantities $\eta / \beta, \beta G$, and $\eta G$. However, these parameters are enough to fully characterize the device. Figure 6 shows the response as a function of wavelength from Fourier Transform Spectrometer (FTS) measurements of individual detectors produced on the same detector wafers as the flight arrays. These are in general agreement with lab measurements done at Cornell using a test dewar to uniformly illuminate the array. The (relative) response curves are useful for determination of the bandpass in computing color corrections. 


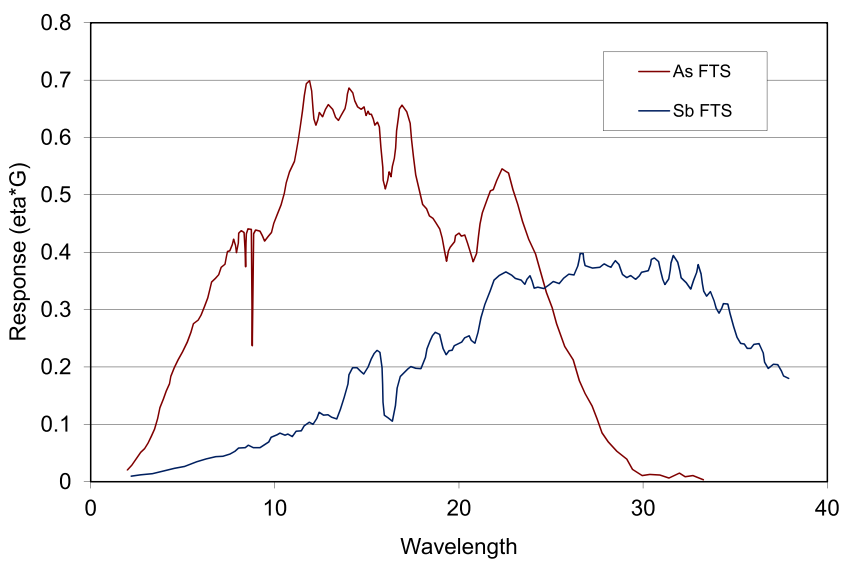

Fig. 6. Responsive quantum efficiency vs. wavelength for the Si:As (brown) and Si:Sb (blue) detector used in FORCAST as determined from FTS measurements of samples from the same detector wafer. These curves are in general agreement with lab measurements of the flight arrays.

Due to the underlying multiplexer, the arrays are subject to a number of artifacts and features including non-linearity, "droop" and "cross-talk." In the mid-infrared non-linearity corrections are driven by the background signal level rather than source signal level. Thus, non-linearities can be significantly mitigated by adjusting the readout rate of the detector to maintain the same integrated background level for all observations. In practice however, low frame rates can increase array noise. This means that the integrated background level cannot be the same for all filters but the level can be fixed for a given filter. Droop causes the output voltages to sag when a signal is present and crosstalk causes bright (or dark) pixels to echo into subsequent pixels sampled on the same output channel. These features and the applied corrections to the data are discussed in Herter et al. (2013).

\section{Electronics}

FORCAST uses standard chopping of the secondary mirror of the telescope to remove short term background variations. Telescope nodding is also used to remove non-common-path background changes introduced by chopping.

\subsection{Requirements}

The high thermal backgrounds present in the midinfrared and the need to optimize detector operations require the electronics to: (1) operate and sample the detector at frame rates up to $500 \mathrm{~Hz}$,
(2) synchronize array frames and data readout with chopping and nodding, (3) have the ability to throw away frames during chop transitions, (4) control the sampling scheme applied to the detector, i.e. destructive reads, non-destructive spinning, sample-up-the-ramp, (5) co-add over multiple chop-nod cycles timescale for nominally $5-10 \mathrm{~s}$ but up to $30 \mathrm{~s}$, and (6) avoid introducing noise into the focal plane, as compared with the nominal device read noise.

Because of the high data rates and the need for flexibility, an FPGA-based (Field Programmable Gate Arrays) hardware approach was taken to handle the data stream in real time.

\subsection{Implementation}

Two FPGA-based boards are used in the system, arranged in a master and slave configuration. The FPGAs are configured at power-up from a local EEPROM and are then reconfigurable at any time over the fiber optic link from the instrument host computer. Clocking information is uploaded to the master FPGA board from the host and resides in a dedicated bank of SRAM. The master FPGA sends array clocking patterns out to level shift cards, which clock the focal planes via cabling inside the dewar. The master FPGA also sends out the convert pulses to 14-bit analog-to-digital converters (ADC). The BIB detectors have 16 outputs, requiring a sample rate of over $4 \mathrm{MHz}$ per output channel, or over $65 \mathrm{M}$ samples/s. Each FPGA board receives pixel data from two quad channel preamp/ ADC boards and the FPGA co-adds the incoming pixel data in local memory. The detector frame rate is controlled by programming a delay counter in the FPGA. The integration time, i.e. how long to continue co-adding frames from the detector before returning the data to the host computer, typically $5-20 \mathrm{~s}$, is controlled simply by setting a counter inside the FPGA.

The system utilizes an Operation Table (OpsTable) which defines at the beginning of every array frame what will happen with that particular read of the detector. The OpsTable specifies if the array will be read destructively or non-destructively, whether this frame will be stored or ignored, where in co-adder memory to place this particular image, and in what chop position the chopping secondary mirror should be. The OpsTable is uploaded into its dedicated SRAM, created inside the FPGA itself. A key feature of the design is 


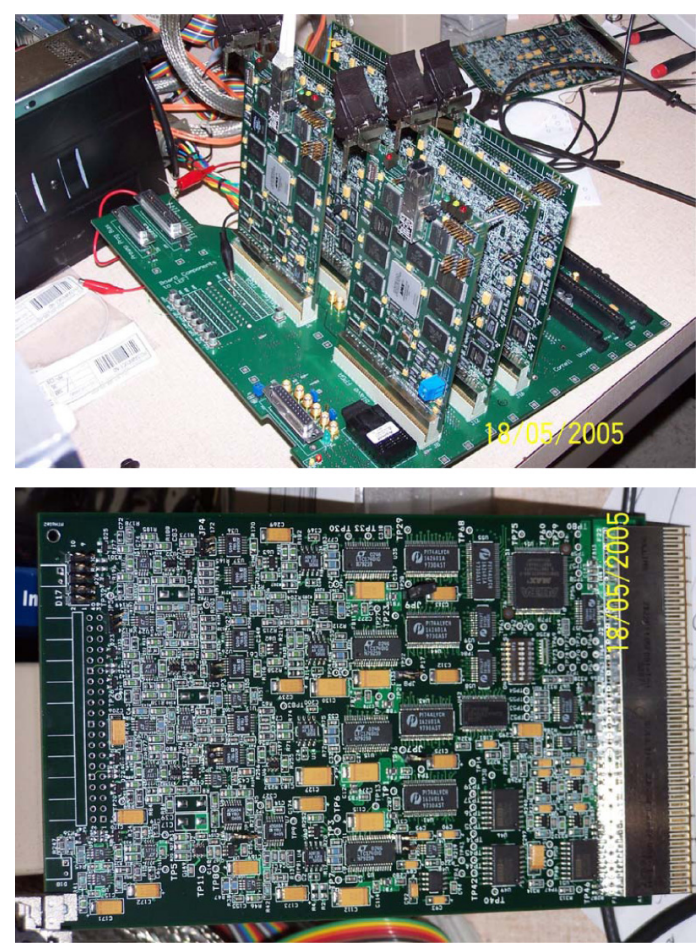

Fig. 7. Photos showing surface-mount electronics boards used in FORCAST. Top: The backplane on the bench, with two FPGA boards and four preamps installed. The extension at the top left of the board is where the signal cable connects the backplane to the dewar. Bottom: The quad channel preamp/ ADC card. Signal flow is from left to right. The array output signals are brought in over the backplane.

that the OpsTable drives the position of the secondary mirror ensuring that the array clocking and sampling of the readout are always phased properly with the chopped signal from the sky.

The FPGA-based hardware, preamp/ADC boards and electronics backplane were designed and fabricated by Cornell. Figure 7 shows the backplane with FPGA controller and preamp/ADC boards installed during a bench test, and single preamp/ ADC board. The backplane is mounted inside an anodized, aluminum box (see Fig. 1), one for each detector. Short cables from the backplane to hermetically sealed connectors through the vacuum wall and then to stainless coax wiring (along with two copper return wires) provide electrical connection to the focal plane. A more detailed discussion of the electronics can be found in Pirger et al. (2006).

\section{Operations and Calibration}

\subsection{Software}

The FORCAST data acquisition and instrument control software provides: (1) an interface for instrument control, (2) interfaces for gathering data from and sending commands to the telescope system interface, (3) instrument data acquisition and storage, (4) remote access to the instrument, and (5) user interfaces (both graphical and text/command based). Instrument control, device drives and related software are written in $\mathrm{C} / \mathrm{C}++$ while the user interface is written in JAVA. The system components provide a high level of computer platform independence and allow remote operation/monitoring/testing/debugging of the instrument. The remote interface allows for relatively low bandwidths since no direct GUI (Graphical User Interface) information is sent, only the data necessary to control the instrument, display status and images. Display functions are handled locally by the Java interface. Data files can be pushed to any network mounted disk.

During normal operations, scripts control data acquisition. Status displays show instrument setting and health, and co-added, chop-nod-subtracted data is continuously displayed to help assess data quality and signal-to-noise ratio.

\subsection{Data acquisition}

For standard science observations FORCAST drives (i.e. chops) the secondary mirror with a square wave at frequencies in the range $1-6 \mathrm{~Hz}$ while the primary mirror of the telescope is nodded every $30-120$ s. In imaging mode, symmetric chopping (secondary mirror oscillating about the optical axis) is used for small $\left(\lesssim 2^{\prime}\right)$ chop throws. The symmetric chop beams have minimal coma due to their relatively small chop throw but the telescope beam becomes increasingly comatic with increasing chop throw. Larger chop throws $\left(2^{\prime}-10^{\prime}\right)$ are performed with asymmetric chopping (one chop beam is on the optical axis, and the other chop beam is far off-axis). The on-axis beam is used to observe the astronomical source because the beam is free of coma. The offaxis beam is used to sample the sky emission only, so the induced coma is irrelevant.

In imaging mode, FORCAST uses an $\mathrm{ABBA}^{(\mathrm{e})}$ nod pattern for symmetric chopping with the chop and nod beams matched (equal throw and opposite directions). An ABAABAABA... nod pattern is used for asymmetric chopping with the nod set to any throw and direction. The same chop and nod

\footnotetext{
${ }^{\mathrm{e}}$ Not to be confused with the $70-80 \mathrm{~s}$ pop/disco music group $\mathrm{ABBA}, \mathrm{A}$ and $\mathrm{B}$ indicate different but repeating nod positions.
} 
patterns are available for spectroscopic mode. In spectroscopic mode, there is an option to nod along the slit regardless of the rotation of field in the FORCAST field-of-view. Dithering can be performed in both imaging and spectroscopic modes.

\subsection{Calibration}

After corrections for non-ideal array performance (Sec. 4.3) and image distortion (Sec. 4.1), photometric calibration is done through measurement of standard stars. Techniques for dealing with nonideal array performance and calibration in general are discussed in detail by Herter et al. (2013).

An issue with calibration is the inability to flat field. Various techniques tried were: (1) the FORCAST calibration box, (2) using the in-flight background signal, and (3) mapping point sources across the array in the lab. All measurements show a 10$20 \%$ response variation over the array but when applied none of these flats reduce the photometric scatter of calibration sources.

For point sources, acquiring data on the same location in the array does show very good reproducibility. ${ }^{(\mathrm{f})}$ For data obtained in this way the calibration uncertainty is about $6 \%$ when using calibration measurements averaged over flight series (Herter et al., 2013).

\section{Performance}

\subsection{Image quality}

The intrinsic image quality of FORCAST can be assessed through lab measurements. Using both an internal pinhole mask at the field stop and external foreoptics to project an image onto the array indicates that the FWHM of the PSF is about $1.5^{\prime \prime}$ at $5.4 \mu \mathrm{m}, 1.1^{\prime \prime}$ from 6.4 to $10 \mu \mathrm{m}$ and diffraction limited $(\mathrm{FWHM}=\lambda / D)$ beyond $10 \mu \mathrm{m}$. Measurements of stars through the telescope with SOFIA parked on the tarmac (in Palmdale, CA) in the relatively transparent $5.4,8.6$, and $11.1 \mu \mathrm{m}$ bands yield similar numbers with FWHM equal to $1.3^{\prime \prime}, 0.9^{\prime \prime}$, and $1.1-1.5^{\prime \prime}$, respectively.

In-flight image quality is influenced mainly by telescope jitter. Figure 8 shows the short-term and long-term PSF at $11.1 \mu \mathrm{m}$ made during a FORCAST observatory commissioning flight (OCF\#3). A stellar source was observed by taking 2500

\footnotetext{
${ }^{\mathrm{f}}$ For extended sources, dithering improves calibration uncertainties by averaging out response variations.
}

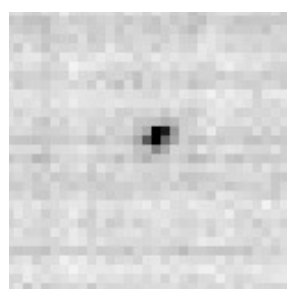

(a)

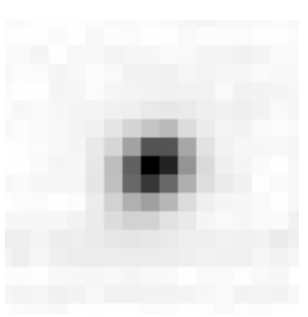

(c)

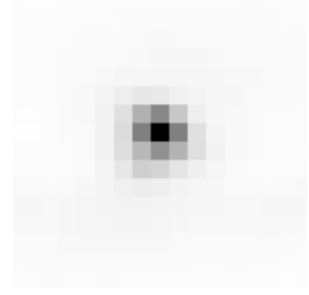

(b)

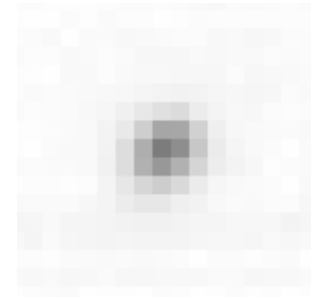

(d)
Fig. 8. Instantaneous and long-term PSF as measured by FORCAST during OCF\#3. Data were taken with the $11.1 \mu \mathrm{m}$ filter of the SWC while sampling at $500 \mathrm{~Hz}$ to acquire 2,500 consecutive frames. Images are: (a) Example single frame exposure, (b) co-addition of images after alignment (FWHM = $\left.1.7^{\prime \prime} \times 1.5^{\prime \prime}\right),(\mathrm{c})$ straight addition $\left(\mathrm{FWHM}=2.6^{\prime \prime} \times 2.1^{\prime \prime}\right)$, and (d) straight addition but with same scale as aligned image.

consecutive frames at $500 \mathrm{~Hz}$. Simply co-adding the data produces a long-term PSF which has a FWHM of $2.6^{\prime \prime} \times 2.1^{\prime \prime}$. Removing jitter by aligning the images before co-adding yields a PSF with $\mathrm{FWHM}=1.7^{\prime \prime} \times 1.5^{\prime \prime} .{ }^{(\mathrm{g})}$ In-flight image quality from four flights during SOFIA Cycle-1d observations of calibration stars is shown in Fig. 9. Further information on SOFIA image quality and plans for improvements can be found in Lampater et al. (2010, 2011); Temi et al. (2014); Graf et al. (2016).

\subsection{Emissivity}

While not directly applicable to science observations, the thermal background plays a pivotal role in determining sensitivity. Nominally, background arises from the sky, the telescope, and the dewar window. The importance of any given source can be wavelength dependent.

With the pupil-viewing lens in the beam, FORCAST views the telescope and its support structure

\footnotetext{
${ }^{g}$ Note that this technique is not applicable to normal observations. A bright infrared source is needed for measuring the centroid and, in general, at this frame rate most observations would become read-noise-limited. In addition, FORCAST is not designed to sustain these data rates.
} 


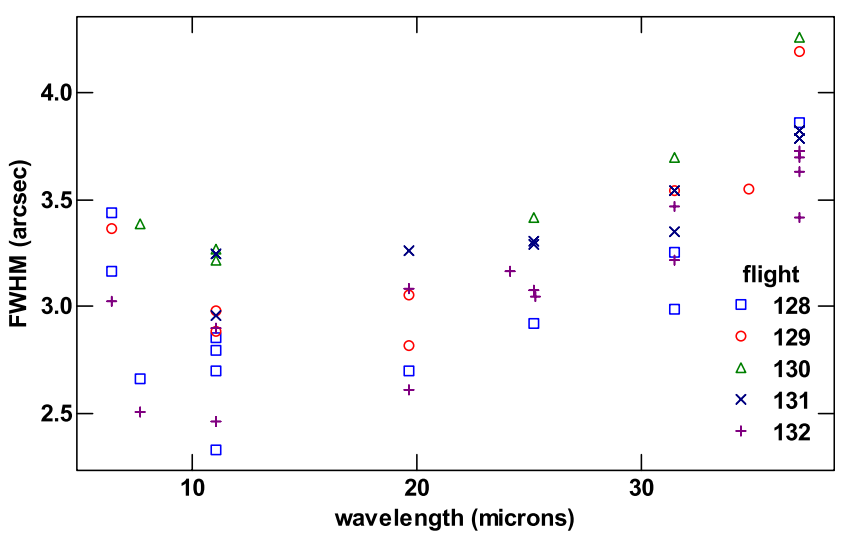

Fig. 9. Example of PSF size and variability over a set of five flights in a series. FWHM of major axis as determined from Cycle-1d observed on SOFIA using $\alpha$ Tau, $\alpha$ Cet, $\beta$ And and $\beta$ Peg. Data for primary temperatures $>-18$ C. Flight 135 has been excluded due to unmatched chop-nods.

(Fig. 10). For the images shown, the reflective secondary button (meant to keep the instrument from seeing the central obscuration of the telescope) is undersized, resulting in a bright ring around the button. Also seen is emission from the secondary support struts reflected off the primary and from the three bolts attaching the button to the center of the secondary mirror. Tipping the collimator to misalign the pupils allows FORCAST to look past the secondary mirror showing both sky and a direct view of one of the support struts.

It is straightforward to estimate the infrared emissivity of the primary-secondary combination using the aligned and misaligned images. Assuming the temperature of all the components are the same, the emissivity of the primary-secondary combination, $\epsilon_{\mathrm{ps}}$ is given by

$$
\epsilon_{\mathrm{ps}}=\frac{S_{\mathrm{sec}}-S_{\mathrm{sky}}}{S_{\mathrm{blk}}-S_{\mathrm{sky}}},
$$
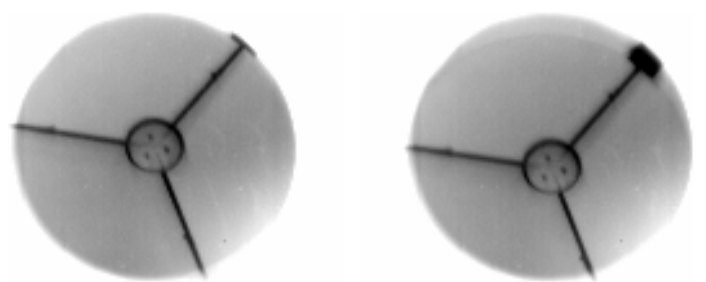

Fig. 10. Left: Image of the pupil at $11.3 \mu \mathrm{m}$ using the pupil viewer mode of FORCAST made with the airplane on the ground. Right: Image with the collimator of FORCAST tipped to misalign the pupils giving a direct view of the sky (on top of image) and a secondary support strut (upper right). See Sec. 7.2 for more details.

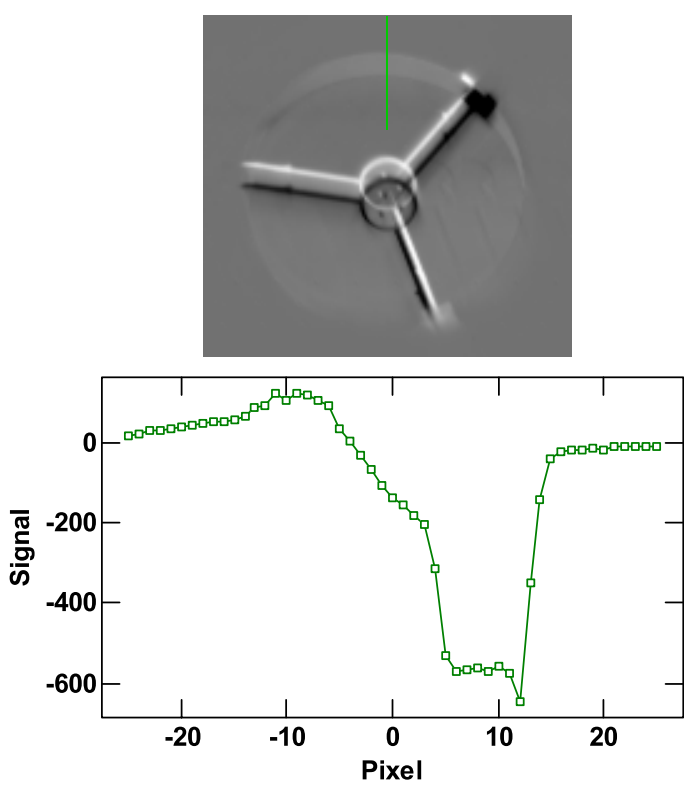

Fig. 11. Top: Difference between misalign and aligned pupil images (Fig. 10). Bottom: Signal along the line shown in the difference image. The depth of the trough in the plot reflects the emissivity of the primary-secondary combination (Sec. 7.2). In the units of the plot, the DC signal on the mirrors is 6580 counts while the DC signal on the exposed strut is 14,700 counts.

where $S_{\text {sec }}$ is the signal looking at the secondary mirror, $S_{\text {sky }}$ is the signal looking off the secondary mirror, and $S_{\mathrm{blk}}$ is the signal from the support strut as viewed past the secondary. The strut is taken to have unity emissivity. Intuitively, one can see that subtracting $S_{\text {sky }}$ removes contributions to the emission from the dewar window and tertiary mirror of the telescope but note that the contributions from the atmosphere also drop out. Figure 11 shows the difference of the two pupil images which is used to estimate $S_{\text {sec }}-S_{\text {sky }}$.

The emissivity of the primary-secondary combination is found to be $\sim 6 \%$. As can be seen in Fig. 11 there is some uncertainty in estimating the baseline for obtaining the difference: 100 counts is approximately $1.5 \%$ of the DC signal. Temperature differences between the structures and non-linearity, particularly for the strut signal, are concerns but additional measurements at different times, frame rates and/or with filters yield similar results, $\epsilon_{\mathrm{ps}}=5.5-7 \%$.

Measurements of the tertiary by FLITECAM ${ }^{(\mathrm{h})}$ indicate an emissivity of $6 \%$. Based on lab tests with FORCAST, the emissivity of the CsI window is

\footnotetext{
${ }^{\mathrm{h}}$ SOFIA Memo SCI-US-REP-OP09-2001 gives estimates of the emissivity of the primary, secondary and tertiary mirrors at $3.08 \mu \mathrm{m}$ based on FLITECAM measurements.
} 
Table 4. FORCAST imaging performance. ${ }^{\mathrm{a}}$

\begin{tabular}{llcccc}
\hline $\begin{array}{l}\lambda_{\text {eff }} \\
(\mu \mathrm{m})\end{array}$ & $\begin{array}{c}\Delta \lambda \\
(\mu \mathrm{m})\end{array}$ & $\begin{array}{c}\text { FWHM } \\
\left({ }^{\prime \prime}\right)\end{array}$ & $\begin{array}{c}\text { FWHM } \\
\left({ }^{\prime \prime}\right)\end{array}$ & $\begin{array}{c}\mathrm{MDCF}^{\mathrm{b}} \\
(\mathrm{mJy})\end{array}$ & $\begin{array}{c}\mathrm{MDCF}^{\mathrm{b}} \\
(\mathrm{mJy})\end{array}$ \\
\hline 5.4 & 0.16 & - & - & 65 & 895 \\
5.6 & 0.08 & - & - & 75 & 285 \\
6.4 & 0.14 & 3.0 & 3.5 & 82 & 92 \\
6.6 & 0.24 & 2.9 & 3.5 & 92 & 121 \\
7.7 & 0.47 & 2.7 & 3.5 & 86 & 104 \\
11.1 & 0.95 & 2.7 & 3.6 & 143 & 155 \\
19.7 & 5.5 & 2.9 & 3.8 & 116 & 123 \\
25.3 & 1.86 & - & - & 236 & 251 \\
8.6 & 0.21 & - & - & 422 & - \\
11.3 & 0.24 & - & - & 320 & - \\
11.8 & 0.74 & - & - & 194 & - \\
24.2 & 2.9 & 3.3 & 4.0 & 154 & - \\
31.5 & 5.7 & 3.4 & 4.3 & 198 & 269 \\
33.6 & 1.9 & - & 4.5 & 414 & 587 \\
34.8 & 3.8 & 3.6 & 4.5 & 285 & 400 \\
37.1 & 3.3 & 3.5 & 4.7 & 383 & 579 \\
\hline
\end{tabular}

Notes: ${ }^{a}$ FWHM for best and nominal worst case with a telescope jitter of $\sim 1.25^{\prime \prime}$ and $\sim 2.1^{\prime \prime}$, respectively.

bMinimum Detectable Continuum Flux (MDCF) to obtain $4 \sigma$ in $900 \mathrm{~s}$ for single (left) and dual (right) channel modes.

$\epsilon_{w}<6 \%$. Thus we expect a total emissivity of $<20 \%$ since at altitude the atmospheric emissivity $<1 \%$ over the $11.3 \mu \mathrm{m}$ filter band. Using the pupil viewer measurements, the total emissivity can be estimated by computing $\epsilon_{\text {total }}=S_{\mathrm{sec}} / S_{\mathrm{blk}}$. For the case given in Fig. 11, this gives $\epsilon_{\text {total }}=0.44$. In-flight measurements typically yield a total emissivity in the $\sim 35 \%$ range. Another estimate of the total emissivity can be made by comparing the DC background signal in normal imaging mode with the signal from stellar calibrators. This gives similar results.

The discrepancy between the individual emissivities and the total has not been resolved. Possible reasons are non-linearities, which are very difficult to assess and correct when the array is partially illuminated or the CsI window is warmer than expected. In-flight, a window temperature of $10^{\circ} \mathrm{C}$ could account for the extra emission, however, this would be $20-30^{\circ} \mathrm{C}$ above ambient which seems extreme since the window is exposed to the ambient atmosphere.

Using measurements of in-flight response from calibrators and removing estimated contributions from the atmosphere, telescope and detectors, the instrument throughput is approximately $10 \%$ for the $19.7 \mu \mathrm{m}$ and longer wavelength filters. However, the detector response changes strongly with bias so our estimates can be influenced by improper normalization of the detector response (shown in Fig. 6). Also, note that this is a "differential" throughput in that the response is measured on top of a much larger background.

\subsection{Sensitivity}

Table 4 gives the nominal FORCAST performance for continuum imaging of point sources. The nominal best and worst case FWHM is given for each wavelength, the Minimum Detectable Continuum Flux (MDCF) for a $4 \sigma$ detection in $900 \mathrm{~s}$ for single change mode, and where applicable the MDCF for dual channel mode.

\section{Sample Science}

The substantial improvement in spatial resolution enabled by SOFIA is best demonstrated through a sample science observation. Figure 12 shows the W3-Main HII region complex imaged with FORCAST during the short science flights (Salgado et al., 2012) and previous measurements at somewhat similar bands. The resolution improvement
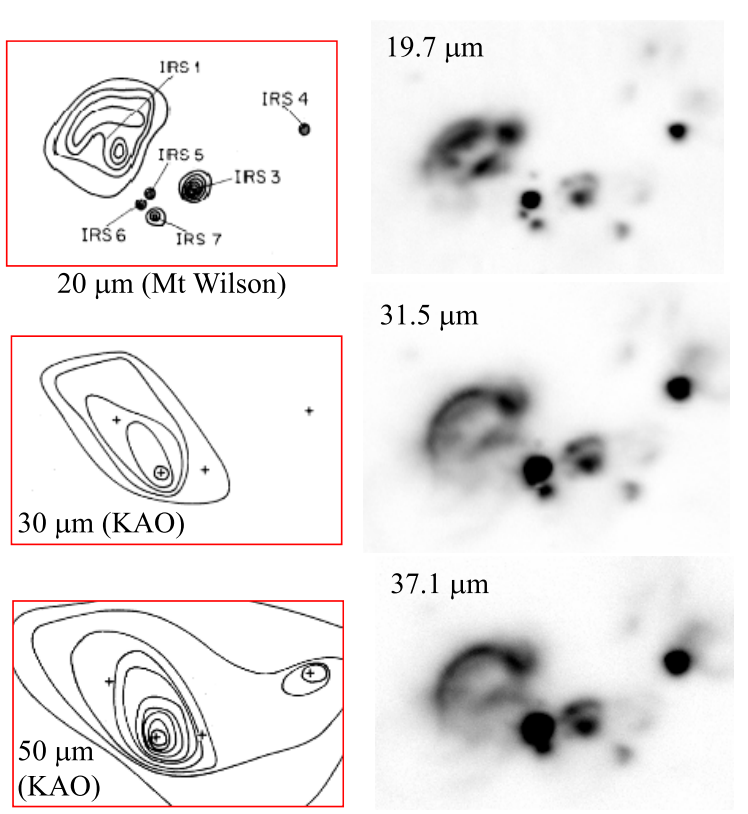

Fig. 12. Comparison of images of the W3-Main HII complex obtained with FORCAST (on the right) at 19.7, 32.5, and $37.1 \mu \mathrm{m}$ (Salgado et al., 2012) on SOFIA along with prior images (on the left) from Mt. Wilson (Wynn-Williams et al., 1972), and the Kuiper Airborne Observatory (Werner et al., 1980). 


\section{T. L. Herter et al.}

over Kuiper Airborne Observatory measurements is, not unexpectedly, dramatic. Comparison to the Mt. Wilson telescope observations, which are comparable in aperture, show a striking enhancement of the structure seen in IRS 1 by FORCAST. More generally, the FORCAST images show considerably more features and structures.

\section{Conclusions}

FORCAST has been a stalwart for SOFIA. The cryostat, cryo-mechanisms, electronics, and software have worked very reliably. It provides capabilities that are not available on any other platform. Its near diffraction-limited spatial resolution and broad wavelength coverage in the mid-IR make it an invaluable tool for studying dust and gas that are heated by UV and optical photons from nearby sources, and the dual-channel mode provides a means for ensuring excellent registration of images in different wavelength bands. An Astrophysical Data System (ADS) search for term "FORCAST" in the text of articles since 2012 (post commissioning) yields 369 publications, 94 of which are referred.

\section{Acknowledgments}

We would like to thank DRS Technologies and Dan Watson (University of Rochester) for providing the spectral response curves shown in Fig. 6 for Si:As and $\mathrm{Si}: \mathrm{Sb}$, respectively. This work is based on observations made with the NASA/DLR Stratospheric Observatory for Infrared Astronomy (SOFIA). SOFIA science mission operations are conducted jointly by the Universities Space Research Association, Inc. (USRA), under NASA contract NAS2-97001, and the Deutsches SOFIA Institut (DSI) under DLR contract 50 OK 0901. Financial support for FORCAST was provided by NASA through award 850098-014 issued by USRA.

\section{References}

Adams, J. D., Herter, T. L., Keller, L. D. et al. [2004] in Proc. SPIE, Vol. 5499, Optical and Infrared Detectors for Astronomy, ed. J. D. Garnett \& J. W. Beletic, pp. 442-451, doi:10.1117/12.551490.

Adams, J. D., Herter, T. L., Gull, G. E. et al. [2010] in Proc. SPIE, Vol. 7735, Ground-based and Airborne Instrumentation for Astronomy III, 77351U, doi:10.1117/12.857049.

Adams, J. D., Herter, T. L., Osorio, M. et al. [2012a] ApJL 749, L24, doi:10.1088/2041-8205/749/2/L24.
Adams, J. D., Herter, T. L., Gull, G. E. et al. [2012b] in Ground-based and Airborne Instrumentation for Astronomy IV. Proc. SPIE, Vol. 8446, article Id. 844616, 11 pp. (2012)., Vol. 8446, doi:10.1117/12.926600.

Arneson, R. A., Gehrz, R. D., Woodward, C. E. et al. [2017] ApJ 843, 51, doi:10.3847/1538-4357/aa75cf.

De Buizer, J. M., Morris, M. R., Becklin, E. E. et al. [2012] ApJL 749, L23, doi:10.1088/2041-8205/749/2/L23.

De Buizer, J. M., Liu, M., Tan, J. C. et al. [2017] ApJ 843, 33, doi:10.3847/1538-4357/aa74c8.

Ennico, K. A., Keller, L. D., Mar, D. J. et al. [2006] in Proc. SPIE, Vol. 6269, Society of Photo-Optical Instrumentation Engineers (SPIE) Conference Series, 62691Q, doi:10.1117/ 12.672256.

Fletcher, L. N., de Pater, I., Reach, W. T. et al. [2017] Icarus 286, 223, doi:10.1016/j.icarus.2016.10.002.

Fuller, L., Lopez-Rodriguez, E., Packham, C. et al. [2016] MNRAS 462, 2618, doi:10.1093/mnras/stw1780.

Gehrz, R. D., Evans, A., Woodward, C. E. et al. [2018] ApJ 858, 78, doi:10.3847/1538-4357/aaba81.

Gordon, M. S., Humphreys, R. M., Jones, T. J. et al. [2018] AJ 155, 212, doi:10.3847/1538-3881/aab961.

Graf, F., Reinacher, A., Jakob, H. et al. [2016] in Proc. SPIE, Vol. 9906, Ground-based and Airborne Telescopes VI, 99061T, doi:10.1117/12.2231803.

Hankins, M. J., Herter, T. L., Maercker, M., Lau, R. M. \& Sloan, G. C. [2018] ApJ 852, 27, doi:10.3847/1538-4357/ aa9cf0.

Harvey, P. M., Adams, J. D., Herter, T. L. et al. [2012] ApJL 749, L20, doi:10.1088/2041-8205/749/2/L20.

Herter, T. [1994] Exp. Astron. 3, 255, doi:10.1007/BF00430173.

Herter, T. L., Hayward, T. L., Houck, J. R., Seib, D. H. \& Lin, W. N. [1998] in Proc. SPIE, Vol. 3354, Infrared Astronomical Instrumentation, ed. A. M. Fowler, 109-115, doi:10.1117/12.317231.

Herter, T. L., Adams, J. D., De Buizer, J. M. et al. [2012] ApJL 749, L18, doi:10.1088/2041-8205/749/2/L18.

Herter, T. L., Vacca, W. D., Adams, J. D. et al. [2013] PASP 125, 1393, doi:10.1086/674144.

Hirsch, L., Adams, J. D., Herter, T. L. et al. [2012] ApJ 757 , 113, doi:10.1088/0004-637X/757/2/113.

Lampater, U., Herter, T., Keas, P. et al.[2010], in Proc. SPIE, Vol. 7733, Ground-based and Airborne Telescopes III, 77330S, doi:10.1117/12.856562.

Lampater, U., Keas, P., Brewster, R. et al. [2011] in Proc. SPIE, Vol. 8336, Integrated Modeling of Complex Optomechanical Systems, 833608, doi:10.1117/12.915762.

Lopez-Rodriguez, E., Fuller, L., Alonso-Herrero, A. et al. [2018] ApJ 859, 99, doi:10.3847/1538-4357/aabd7b.

Materese, C. K., Bregman, J. D. \& Sandford, S. A. [2017] ApJ 850, 165, doi:10.3847/1538-4357/aa960d.

Nikola, T., Herter, T. L., Vacca, W. D. et al. [2012] ApJL 749, L19, doi:10.1088/2041-8205/749/2/L19.

Pirger, B. E., Schoenwald, J., Herter, T. L. et al. [2006] in Proc. SPIE, Vol. 6276, Society of Photo-Optical Instrumentation Engineers (SPIE) Conference Series, 62760X, doi:10.1117/ 12.672249 .

Salgado, F., Berné, O., Adams, J. D. et al. [2012] ApJL 749, L21, doi:10.1088/2041-8205/749/2/L21.

Shuping, R. Y., Morris, M. R., Herter, T. L. et al. [2012] ApJL 749, L22, doi:10.1088/2041-8205/749/2/L22. 
Temi, P., Marcum, P. M., Young, E. et al. [2014] ApJS 212, 24, doi:10.1088/0067-0049/212/2/24.

Werner, M. W., Becklin, E. E., Gatley, I. et al. [1980] ApJ 242, 601, doi:10.1086/158496.

Wynn-Williams, C. G., Becklin, E. E. \& Neugebauer, G. [1972] MNRAS 160, 1, doi:10.1093/mnras/160.1.1.
Young, E. T., Becklin, E. E., Marcum, P. M. et al. [2012] ApJL 749, L17, doi:10.1088/2041-8205/749/2/L17.

Zhang, Y., Tan, J. C., De Buizer, J. M. et al. [2013] ApJ 767, 58, doi:10.1088/0004-637X/767/1/58. 